\title{
Calculation of the auxiliary buffers time with regard to the robustness of the construction schedule to disruption
}

\author{
Mieczysław Połoński ${ }^{1, *}$ \\ ${ }^{1}$ Warsaw University of Life Sciences (WULS - SGGW), Faculty of Civil and Environmental Engineering, \\ ul. Nowoursynowska 159, 02-787 Warsaw, Poland
}

\begin{abstract}
The literature offers numerous proposals for calculating the project buffer, located at the end of the critical chain. However, the structure of construction schedules, often consisting of several dozen or several hundred tasks, requires planning a larger number of buffers, also located on the critical chain. The article proposes an original method of dividing the duration of one buffer located at the end of a protected chain (e.g. a project buffer) into several auxiliary buffers, protecting individual fragments of this path. The basis of the algorithm that calculates their size is to take into account the robust of protected task paths before the deadline for their completion is disrupted. As a measure of robust understood in this way, the ratio of the buffer size to the sum of the variance of the task duration of the protected draft was applied. The criterion of searching for the optimal time division into several buffers was to maximize the minimum robust of all analyzed paths. The application of the proposed optimization model is illustrated on the example of a specific construction schedule.
\end{abstract}

\section{Introduction}

When E. Goldratt proposed that the project buffer (PB) and feeding buffers (FB) be placed in the structure of network schedules [1], many authors attempted to develop a method for determining their size [2-4]. This was mainly due to the fact that Goldratt in his book gave only general assumptions, without indicating the exact method of their calculation. Two different approaches to the problem of time reserve can be found in the literature. The first one, according to Goldratt's proposal, assumes the introduction of several time buffers located in selected places of the network depending on the schedule being prepared. The second solution, instead of creating separate buffers, indicates the possibility of adding a time reserve to each task in the schedule, thus increasing their initial duration.

Both proposed solutions assume a slightly different approach to the issue of robustness of the drawn construction schedule for disruption. The first puts the main emphasis on keeping the planned deadline, allowing for the possibility of exceeding some of the deadlines for particular tasks, which make up the entire schedule. In the second approach, the main importance is attached to meeting the planned dates of all tasks, trying to prevent any delays. This is usually motivated by the necessity of keeping in agreements concluded with individual subcontractors of scheduled dates of works, or limited access to selected resources necessary to perform tasks. However, observing the construction market, it can be seen that in the majority of cases, the main problem is to meet the deadline for the entire project. Sometimes, when the planned investment is large, it can be divided into stages. Then, at least some of the dates of its completion should be subject to special protection, especially if they are protected by the constrain date.

However, regardless of whether the date-constraints have been defined or not, the tasks whose date should be particularly protected usually lie in the critical chain and do not have slack time. Therefore, in the critical chain should be located several buffers (referred to by the author as the auxiliary buffers - $\mathrm{AB}$ ), and not just one, project buffer $(\mathrm{PB})$, as proposed by Goldratt. This will allow better protection of key tasks (e.g. milestones), facilitate project management and allow more effective monitoring of the use of individual buffers during implementation. In order to apply such a solution, however, we must know how to allocate the time reserve provided for the whole project to individual auxiliary buffers, since each of them lies on the critical chain, protects a certain path of tasks and has an impact on the completion date of the entire construction entity.

Since the application of time buffers in the literature, a number of suggestions on how to calculate them can be found, both for entire schedules or fragments protected by individual buffers [5-7] as well as individual tasks [8]. The proposed methods differ among others in the following assumptions and solutions:

- task duration are defined as deterministic or probabilistic values (also as fuzzy numbers with different membership functions),

- in the case of probabilistic data, a different type of task duration distribution is assumed (usually normal, beta, PERT, lognormal, triangular),

- the output task duration is shortened after inserting buffers or remains unchanged, 
- after inserting buffers into the structure of schedules it is allowed to change the course of the critical chain or its original position is protected,

- different robustness criteria to disruption are adopted [9].

The article proposes an original method for dividing the time of one project buffer located at the end of the protected chain into several, protecting individual fragments of this chain (e.g. auxiliary buffers). The presented method is aimed at increasing the robust of the schedule to disruption, and concerns the protection of selected paths of tasks completed with individual buffers, and not each task separately. The concept of the location of buffers and their types in construction schedules was taken from the MP-KP method [10], however the method of determining their time has been modified and adapted to the concept of robustness of the schedule aimed at keeping the deadline (so-called quality robustness [9]).

\section{Basic information about auxiliary buffers}

Construction schedules count several dozen or several hundred tasks, have a complex structure and their critical chain often diverges. All this makes the use of only two types of buffers (PB and FB) insufficient to fully protect such schedules. As part of the MP-KP method, whose the author is a co-creator, their new types have been proposed. The most important of these are auxiliary buffers (AB). They are placed in the critical chain immediately before joining with another, non-critical path, or before a task whose deadline requires special protection (e.g. milestone) [10].

When planning the date of completion of works, two related issues are of fundamental importance. The first is the stage of the investment process, during which we prepare the schedule. The second is the fact whose point of view we consider it from. It may be an investor who is trying to estimate the real time for the planned investment before signing the contract, or a contractor who has already concluded a contract for the execution of construction works and has a deadline to complete the works. It is worth noting that although the methods found in the literature are based on different assumptions, they almost always rely on the determination of buffer time in the first place, and only in the next on the calculation of the end date of the entire project, taking into account the designated buffers (the attempt to calculate the probability of keeping such a deadline is relatively rare). Such solution may satisfy the investor, but most often it is not acceptable by the contractor who constructs the schedule, ensuring that the deadlines are met without charging contractual penalties for delaying the completion of works. For this reason, in the MP-KP method, the size of each time buffer $\left(T B_{j}\right)$ (regardless of whether it is a project buffer, auxiliary or feeding) is determined as the difference between $T_{P}$ termination of the whole chain protected by buffer of $n$ tasks and the sum of critical chain task duration $t_{i}^{s h r}$ [10]. The duration $t_{i}^{s h r}$, according to Goldratt's assumption, most often shortens in relation to the initial time. The term $T_{P}$ is calculated on the basis of the central limit theorem with the assumed probability of reaching the final date $P$ (or the adopted constraint deadline). Its calculation requires knowledge of the variance of tasks in the protected path.

$$
T B_{j}=T_{p}-\sum_{i=1}^{n} t_{i}^{s h r}
$$

The calculation formula adopted in this way gives the project manager a wide range of possibilities, as she or he can freely set the type of task duration distribution, $\mathrm{P}$ probability of completing the desired deadline for completing the entire $T_{P}$ critical chain and shortening the duration of each task (taking into account the existing technological and organizational constraints). The last parameter $\left(t_{i}^{s h r}\right)$ is assumed to be either a characteristic value of the time distribution (e.g. optimistic time in the PERT distribution), or calculated as a quantile with assumed probability level $p$ time of a single task (it is necessary to distinguish $P$ as the probability of keeping the entire critical chain, and $p$ as probability keeping a single task). It should also be noted that the total time of truncated tasks in the chain and buffer protecting it ensures that the accepted term of termination $T_{P}$ is reached with assumed probability $P$, i.e. the shorter task duration is adopted, the buffer time may be larger.

\section{3 . Buffers sizing approaches}

The method of calculating buffers proposed in the article is based on the following assumptions:

- we have three assessments of the execution time of individual tasks in the schedule, pessimistic, optimistic and the most probable,

- we can set up the time task distribution and on the basis of the estimated three time estimates, calculate its parameters, i.e. variance and any quantile,

- the deadline for completing the entire buffer-protected task chain can be determined as the constrain date (earlier or later than the initially calculated schedule end date without taking into account the buffers) or calculated on the basis of the assumed probability of its keeping,

- depending on the assumed deadline for completing the task path, task duration may (but do not have to) be shortened in relation to duration in the schedule without buffers,

- time buffers are separate elements of the schedule and protect selected paths of tasks and are not a part of the duration of individual tasks,

- the number and location of individual buffers in the schedule structure is determined in accordance with the assumptions of the MP-KP method,

- every task in the schedule is assigned to the bufferprotected path.

As it has been previously written, in the case of large, complex schedules of construction works, there is a justified need to deploy more buffers in the critical chain. They are called auxiliary buffers (AB) and, like 
all other buffers, they need to be calculated for their duration. Since, regardless of the type of buffer being analysed and the number of jobs in the chain protected by this buffer, the same calculation formula is used, it causes a kind of conflict with these buffers. The essence of this problem is described below in a very simple example.

Let's assume, for simplicity, that the critical chain has 60 tasks and each task in this chain has the same average time $d_{i} 10$ days and standard deviation $\delta_{i}$ equal to 1 . Let's also assume that these values were calculated based on three time estimates and assumed type of task time distribution (e.g. PERT or triangular). To calculate the probability of completing the chain $T_{P}$ consisting of all of tasks at the assumed probability level, we can use the central limit theorem. On its basis, we assume that the distribution of the sum of independent random variables (of any distribution) approaches the normal distribution as the number of these random variables increases, so $T_{P}$ can be calculated from the formula:

$$
T_{P}=\sum_{i=1}^{k} d_{i}+U_{P} \times \sqrt{\sum_{i=1}^{k} \delta_{t i}^{2}}
$$

where $U_{P}$ is the distribution value of the normal standard distribution $\mathrm{N}(0,1)$ for the probability $P, k$ - the number of considered activities in the chain, and $d_{i}$ the average value of the time of activity $i$.

Assuming, for example, $\mathrm{P}=0.97$ and substituting for the above formula, we get:

$T_{0.97}=\sum_{i=1}^{k 60} 10+1.88 \times \sqrt{\sum_{i=1}^{60}\left(60 \times 1^{2}\right)=614.6}$

This result indicates that by assuming $d_{i}$ task times in the schedule, the project buffer located at the end of this chain should be 14.6 days long. Suppose, however, that we want the whole chain of 60 tasks not to be protected with only one buffer at its end, but buffers occurred after every 20 tasks, i.e. in total, we plan 3 buffers. The deadline for completing a chain of 20 tasks is:

$T_{0.97}=\sum_{i=1}^{k 20} 10+1.88 \times \sqrt{\sum_{i=1}^{20}\left(20 \times 1^{2}\right)}=208.4$

so the buffer after every 20 tasks should be 8.4 days (with the same task duration). However, then the total time accumulated in buffers will be $3 * 8.4=25.2$ days, and the makespan will be shifted to 625.2 days (it corresponds to the probability of keeping $P$ equal to 0.999). The difference in the sums of times accumulated in buffers lying in the same chain depending on their number results from a simple rule of mathematics, which states that the sum of roots is not equal to the root of the sum. As you can see, if the calculations were left at this stage, the end date of the entire project would depend on the number of planned buffers, which is not acceptable.
In order to obtain the correct result, the times of these buffers should be reduced so that their sum corresponds to the time of one project buffer. To achieve this, it is necessary to set a rule according to which such a reduction will be made. And here we come to the heart of the considered issue, that is, to adopting a method of shortening the pre-calculated values of auxiliary buffers. So far, in the MP-KP method, this was done in proportion to the size of the originally determined buffers, i.e. the larger the buffer, the greater the shortening. The author proposes to accept a different rule, related to the robust scheduling.

The concept of robust scheduling in terms of its robustness to disruption has been widely described, among others by Herroelen and Leusa [9]. Seeing that, as it was written in the introduction, in the majority of cases the main problem in the construction industry is to meet the deadline of the entire project, in the article the author focused on quality robustness. The literature provides the proposed measures of robustness and the possibility of its use to determine the size of buffers. For example, Al-Fawzan [11] defines the measure of robustness of the schedule as the sum of free floats of all its tasks. Kobylański and Kuchta in their article [8] criticize this solution and propose that the robustness of a schedule can be measured by the minimum of the ratios (calculated for all the activities) free slack / durations. The measure defined in this way has, among other things, the advantage of allowing to build a model for optimizing the stock of free tasks. An interesting proposition is an attempt to determine the value of expected delays of processes in relation to those planned using the Monte Carlo simulation methods [12], however, such solution requires the construction of a model and simulation calculations separately for each analysed schedule, which limits its application.

The solution proposed by the author concerns the determination of the time of individual $\mathrm{AB}_{\mathrm{j}}$ auxiliary buffers, when their sum is known, and the size of individual buffers is calculated taking into account the robustness to disruption of task paths protected by these buffers. As a measure of chain robustness, the author proposes to take the ratio of the $A_{\mathrm{j}}$ buffer to the sum of the time variance $\delta^{2}$ of all critical tasks of the protected path. Because the variance of the task time indicates the uncertainty of estimating its time, the adoption of such a measure should be considered justified. On the other hand, in the optimization model, which will be used to calculate all buffers, the maximization of the minimum value $\mathrm{AB}_{\mathrm{j}}$ / (sum of $\delta_{i}{ }^{2}$ ) was assumed to be the criterion.

$z=\min \left(A B_{j} / \sum \delta_{i}^{2}\right) \rightarrow \max$

with limitations:

Sum $\mathrm{AB}_{\mathrm{j}}=\mathrm{BP}$ where $j$ is the number of $\mathrm{AB}$ buffers located in the critical chain.

The proposed model does not meet the conditions of linearity, but its solution does not cause difficulties with available programs (e.g. LINGO), or the Solver tool in MS Excel, using the GRG nonlinear algorithm or evolutionary algorithm. 
The fact that the criterion applied by the author is better than that proposed by Kobylański and Kuchta can be proved by a simple example. Let us assume that the critical chain consists of two successive task paths, each of which ends with a buffer, respectively labelled $\mathrm{AB}_{1}$ and $\mathrm{AB}_{2}$. The first chain (completed with the $\mathrm{AB}_{1}$ buffer) counts only three tasks, the second (completed with $\mathrm{AB}_{2}$ buffer) fifteen tasks. Let us also assume that the times of all tasks are described by the triangular distribution and three time estimates $\mathrm{A}, \mathrm{B}$ and $\mathrm{C}$ are estimated as the shortest, longest and most likely times. In the first chain all tasks have a time $\mathrm{C}=10$ and times $\mathrm{A}$ and $\mathrm{B}$ are away from $50 \%$ of time $\mathrm{C}$, that is respectively 5 and 15 . Then the variance of each of these tasks $\delta_{i}{ }^{2}$ equals 4.17 and their sum in the chain is 12.50 . In the second path, all tasks have a time $\mathrm{C}=2$ and times $\mathrm{A}$ and $\mathrm{B}$ are from this assessment also $50 \%$ of time $\mathrm{C}$, i.e. equal to 1 and 3 respectively. The variance of each of these tasks $\delta_{i}{ }^{2}$ is then equal to 0.17 , and their sum 2.50. It is easy to notice that the sum of the most probable $\mathrm{C}$ times in both paths is the same and amounts to 30 . By calculating the sizes of these buffers according to the formula proposed in the article, they will be 16.7 for $\mathrm{AB}_{1}$ and 3.3 for $\mathrm{AB}_{2}$. On the other hand, if the ratio of the size of the buffer to the time of the protected chain (as proposed by Kobylański and Kuchta) is the measure of robustness, the size of both buffers would be the same, that is $A B_{1}=A B_{2}=10$. The fact that the buffer sizes should be different may be indicated by the calculated date of completion of these paths at the assumed probability level $P=0.97$. It is for the first chain round 43 and for the second one 31 . As you can see, the first chain should have a much larger buffer than the second one, which is consistent with the results proposed in the article.

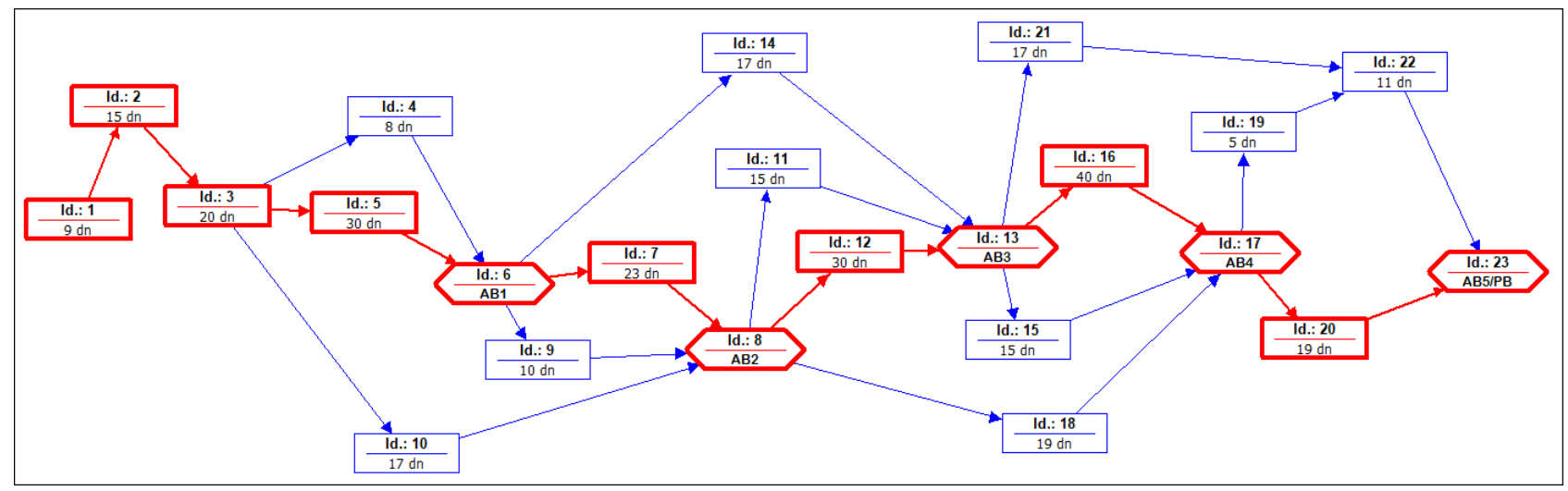

Fig. 1. The relationship network with the location of feeding buffer on the critical chain

\section{Calculation example}

An exemplary schedule for the implementation of a construction project was taken from Skorupka's paper [13]. The method proposed in the article is useful mainly when planning large building objects, when the schedules count even several hundred tasks. However, the example given below has only 18 tasks, which allows to quote all the data necessary for calculations and to track their results. The schedule concerns the construction of a shopping, service and office centres. The building was designed as a 2-segment, 4-floor, without basement with two staircases and two external elevators. The building area is $1386 \mathrm{~m}^{2}$, the cubature of $17589 \mathrm{~m}^{3}$. The task duration is described by the triangular distribution, giving the three characteristics $\mathrm{A}$, $\mathrm{B}$ and $\mathrm{C}(\mathrm{A} \leq \mathrm{C} \leq \mathrm{B})$ (Table 1). Assuming $\mathrm{C}$ time as estimation of task duration, the total duration of works was calculated as 185 working days, and non-bifurcated critical chain was carried out for 8 tasks. Five AB auxiliary buffers are located on it, the last of which at the end of this chain is also a project buffer (Fig. 1).

Based on the parameters of the critical chain task duration and assumed probability of completion of the entire undertaking $\mathrm{P}$ equal to 0.97 from the Central limit theorem, the execution time was 209 days. Assuming task duration such as in the starting schedule, the total time that can be spent on all five buffers is then 209 $185=24$ days. Next, the values of individual buffers were calculated in accordance with the proposed formula (rounded to the integer values). The results are shown in table 2.

Table 1. Duration and variance of critical chain tasks - which buffer protects the ID task.

\begin{tabular}{|c|c|c|c|c|c|c|}
\hline No & ID & $\begin{array}{c}\text { AB } \\
\text { No }\end{array}$ & $\begin{array}{c}\text { Time } \\
\mathrm{A}\end{array}$ & $\begin{array}{c}\text { Time } \\
\mathrm{C}\end{array}$ & $\begin{array}{c}\text { Time } \\
\mathrm{B}\end{array}$ & $\begin{array}{c}\text { Variance } \\
\delta_{i}{ }^{2}\end{array}$ \\
\hline 1 & 2 & 3 & 4 & 5 & 6 & 7 \\
\hline 1 & 1 & $\mathrm{AB} 1$ & 7 & 10 & 12 & 1,06 \\
\hline 2 & 2 & $\mathrm{AB} 1$ & 14 & 15 & 20 & 1,72 \\
\hline 3 & 3 & $\mathrm{AB} 1$ & 19 & 20 & 24 & 1,17 \\
\hline 4 & 5 & $\mathrm{AB} 1$ & 28 & 30 & 39 & 5,72 \\
\hline 5 & 7 & $\mathrm{AB} 2$ & 21 & 23 & 30 & 3,72 \\
\hline 6 & 12 & $\mathrm{AB} 3$ & 29 & 30 & 37 & 3,17 \\
\hline 7 & 16 & $\mathrm{AB} 4$ & 38 & 40 & 51 & 8,17 \\
\hline 8 & 20 & $\begin{array}{c}\mathrm{AB} 5 / \\
\mathrm{PB}\end{array}$ & 18 & 19 & 24 & 1,72 \\
\hline
\end{tabular}


Table 2. The results of calculating the size of buffers for the project completion date of 209 days.

\begin{tabular}{|c|c|c|c|c|}
\hline Buffer & $\begin{array}{c}\text { Buffer } \\
\text { duration }\end{array}$ & $\sum \delta_{i}^{2}$ & $\mathrm{AB} / \sum \delta_{i}^{2}$ & $\begin{array}{c}\text { Objective } \\
\text { func. }\end{array}$ \\
\hline $\mathrm{AB} 1$ & 8 & 9,67 & 0,83 & \multirow{5}{*}{0,83} \\
\hline AB2 & 4 & 3,72 & 1,07 & \\
\hline $\mathrm{AB} 3$ & 3 & 3,17 & 0,95 & \\
\hline $\mathrm{AB} 4$ & 7 & 8,17 & 0,86 & \\
\hline $\begin{array}{l}\text { AB5/ } \\
\text { PB }\end{array}$ & 2 & 1,72 & 1,16 & \\
\hline$\sum$ & 24 & & & \\
\hline
\end{tabular}

\section{Conclusion}

The article proposes an original method of dividing the time of one buffer located at the end of a protected chain (e.g. a project buffer) into several auxiliary buffers, protecting individual fragments of this path. This will allow more sufficient protection of key tasks (e.g. milestones), facilitate project management and allow more effective monitoring of the use of individual buffers during the implementation of the investment. The basis of the algorithm calculating the size of these buffers is to take into account the robustness of paths of protected tasks before the term of their completion is outdated. As a measure of robustness understood in such a way, the ratio of the buffer size to the sum of variance of task duration of the protected chain was adopted, and the criterion of searching for the optimal buffer time division was to maximize the minimum robustness of all analysed paths. The proposed optimization model does not meet the conditions of linearity, but its solution with available programs is not difficult. The attached example allows to analyse the proposed calculation method and indicates the improvement of the robustness of the schedule so designed.

\section{References}

1. E. M. Goldratt. Critical chain, Great Barrington, MA: North River Press, (1997)

2. R. C. Newbold, Project Management in the Fast Lane: Applying the Theory of Constraints, St. Lucie Press, New York, (1998)

3. L. P. Leach, Critical Chain Project Management, Artech House, Boston, (2000)

4. W. Herroelen, R. Leus, European Journal of Operational Research, 165: 289-306, (2005)

5. O.I. Tukel et al., European Journal of Operational Research, 172: 401-416, (2005)

6. L. Bie, N. Cui, X. Zhang, International Journal of Production Research, 50(24): 7343-7356, (2012)

7. M. Połoński, A. Ziółkowska, Scientific Review Engineering and Environmental Sciences, 3(45): 2634, (2009)

8. P. Kobylański, D. Kuchta, International Journal of Production Economics, 107(2): 496-501, (2007)
9. W. Herroelen, R.Leus, International Journal of Production Research, 42(8): 1599-1620, (2004)

10. M. Połoński, K. Pruszyński, Przegląd Budowlany, 2: 45-49, (2008)

11. M.A. Al-Fawzan, M. Haouari, International Journal Production Economics, 96: 175-187, (2005)

12. P. Jaśkowski, S. Biruk, Technological and Economic Development of Economy, 17(3): 429444, (2011)

13. D. Skorupka, Metoda identyfikacji i oceny ryzyka realizacji przedsięwzięć budowlanych, Wojskowa Akademia Techniczna, Poland, (2007) 